\title{
Sublimidad estética y ascetismo burgués: A propósito de la "Analytik des Erhabenen"
}

\begin{abstract}
This paper investigates the disproportion of the Analytics of the Sublime in context of the Critique of Judgement, as an analogy to the impossibility of reconciling moral theory and practice, nature and reason; thus the bridge between the first and the second critiques, which should be mediated by the third, is marked anew: the sublime corresponds to the violence masked within social processes. Kant's position is worked out upon the background of Shaftesbury and Burke and thus emerges the fact that the Königsberg philosopher is oriented towards ahistorical rational ideas. His concept of the sublime (as well as his ethics) is therefore situated in the traditions of protestant ascetism and bourgeois-capitalism. Both the beholder of sublime nature and art and the follower of moral imperative must equally relinquish everything material and the direct satisfaction of their yearnings in favour of a higher, intellectual satisfaction. In the same way, the absence of form or measure of the sublime has its parallel in the "negative infinity" of capital and in technological "second nature".
\end{abstract}

El autor es Profesor Adjunto de Literatura Alemana en la Universidad de Buenos Aires. 
Keywords: Kant; Sublime; Bourgeois Asceticism; Dialectic of Enlightenment. Zusammenfassung: Der Artikel untersucht die Disproportion der Analytik des Erhabenen im Rahmen der Kritik der Urteilskraft, die analog für das nicht Versöhnbare in Theorie und moralischer Praxis / Natur und Vernunft steht und damit die Brüche zwischen der ersten und zweiten Kritik erneut markiert, die von der dritten Kritik gerade vermittelt werden sollten: Das Erhabene entspricht der verschleierten Gewalt in den gesellschaftlichen Prozessen. Kants Position wird vor dem Hintergrund von Shaftesbury und Burke herausgearbeitet und dabei der Akzent auf die Orientierung des Königsbergers an ahistorischen Vernunftideen gelegt. Sein Begriff des Erhabenen (ebenso wie seine Ethik) steht damit in der Tradition der protestantischen wie der bürgerlich-kapitalistischen Askese. Für den Betrachter der sublimen Natur und Kunst wie für den, welcher den moralischen Imperativ befolgt, gilt gleichermaßen die Zurückstellung des Materiellen und der direkten Trieberfüllung zugunsten einer höheren, geistigen Befriedigung. Entsprechend findet die Form- und Maßlosigkeit des Sublimen ihre Parallele in der ,schlechten Unendlichkeit“ des Kapitals und der technologischen „zweiten Natur“.

Stichwörter: Kant; Erhabenes; bürgerliche Askese; Dialektik der Aufklärung. Resumo: $\mathrm{O}$ artigo examina a desproporção da analítica do sublime no âmbito da crítica do julgamento como analogia à impossibilidade de conciliar teoria e prática moral / natureza e razão. Com isso, delineia-se novamente a ponte entre a primeira e a segunda críticas, as quais devem ser mediadas pela terceira: o sublime corresponde à violência velada nos processos sociais. A posição de Kant é trabalhada contra o pano de fundo de Shaftesbury e Burke e, assim, a tônica recai sobre a orientação do filósofo de Königsberg em relação a idéias racionais ahistóricas. Seu conceito de sublime (assim como sua ética) posiciona-se, portanto, na tradição do ascetismo protestante assim como do capitalista-burguês. Tanto o observador da natureza e arte sublimes como aquele que segue o imperativo moral devem igualmente preterir o que é material e a satisfação direta dos anseios em favor de uma satisfação maior, intelectual. Do mesmo modo, a ausência de forma e medidas do sublime encontra seu paralelo na „infinidade negativa“ do capital e na „segunda natureza“ tecnológica.

Palavras-chave: Kant; o sublime; ascetismo burguês; dialética do iluminismo. 


\section{La marginalidad de lo sublime}

Un detalle llamativo en la Kritik der Urteilskraft (1790) es el exiguo lugar concedido a la investigación de lo sublime. La "Analítica de lo sublime" ocupa cerca de la mitad del espacio acordado al análisis de la belleza; y la desproporción se acentúa por el hecho de no hallarse lo sublime en relación armónica con el juicio de gusto. A la pregunta de por qué se ha reservado para lo sublime un lugar tan subsidiario dentro de la economía de la tercera crítica, podría responderse apuntando que dicha experiencia no contribuye al propósito general de la obra, es decir: a la reconciliación del conocimiento teórico con la praxis moral, o - dicho en otros términos - de la naturaleza con la razón. Marca de diferendo y oposición, lo sublime reabre las escisiones entre la primera y la segunda críticas que se había intentado subsanar a través del juicio de gusto. El propio Kant destaca que el confinamiento de la teoría de la sublimitas al papel de "mero apéndice" del análisis del juicio de gusto, responde a que las ideas de lo sublime están separadas del principio de la finalidad de la naturaleza; y en la postulación de dicho principio se fundaba la aptitud de la capacidad de juzgar para establecer una mediación entre la filosofía teórica y la práctica. Según se afirma en la "Introducción" a la Kritike der Urteilskeraft

Das, was diese a priori und ohne Rücksicht auf das Praktische voraussetzt, die Urteilskraft, gibt den vermittelnden Begriff zwischen den Naturbegriffen und dem Freiheitsbegriffe, der den Übergang von den reinen theoretischen zur reinen praktischen, von der Gesetzmäßigkeit nach der ersten zum Endzwecke nach dem letzten möglich macht, in dem Begriffe einer Zweckmäßigkeit der Natur an die Hand (KANT 1990: \IX, 34).

La idea de una finalidad en la naturaleza suscita la ilusión de una realidad material "humanizada" o, al menos, inteligible en términos humanos: los paisajes hermosos parecen hablar figuradamente, se dirigen a nosotros en una escritura cifrada que imaginamos urdida para cautivarnos, aun cuando su sentido no cese de resultar enigmático. Por esta capacidad del paisaje para adaptarse a la subjetividad, la naturaleza aparece ante nuestros ojos, en lo bello, como aquel sitio en el que podemos descubrir un hogar genuino: en su plenitud de formas, los escenarios naturales bellos 
despiertan la ilusión de haber sido creados para el hombre; a nuestra contemplación desinteresada parecen devolver - como en la conocida imagen baudelaireana - sus regards familiers. Nada de esto ocurre en lo sublime: la naturaleza salvaje nos amenaza y repele, y busca demostrar con ello que nuestro destino se encuentra en una esfera distinta de la sensible; en este sentido, la definición de la "Analítica de lo sublime" como un cuerpo extraño dentro de la Kritik der Urteilskraft es tan legítima como su tradicional caracterización en cuanto punto de transición entre lo sensible y lo suprasensible. La experiencia de la sublimidad consigue casi trascender el ámbito de la estética y elevarse hacia el libre ámbito de la razón, hacia la moralidad, para la cual aquella provee un analogon, en la medida en que el bien moral, según afirma Kant, no debe ser representado como bello, sino como sublime ${ }^{1}$.

Pero subsiste la pregunta sobre si la marginalidad de la "Analítica" es producto de la indiferencia frente a esa experiencia estética, o si no deberá verse en ella, por el contrario, la confirmación de la superioridad de lo sublime frente a la belleza. De acuerdo con ciertos intérpretes, Kant se vio compelido a incluir en la Kritik der Urteilskraft una "Analytik des Erhabenen" solo a causa del interés suscitado por la sublimitas en el pensamiento y en la sensibilidad contemporáneas, aun cuando el filósofo sería consciente de la imposibilidad de conciliar las cualidades de lo sublime con las conclusiones extraídas del análisis del juicio de gusto (y, por ende, con la tercera crítica en general) ${ }^{2}$. Esta versión contrasta con el énfasis puesto por Lyotard, Nancy o LacoueLabarthe en destacar la importancia del análisis de lo sublime, no ya en el marco de la Kritik der Urteilskraft, sino en la evolución de la estética moderna. En particular, Lyotard ha dedicado un trabajo de años al comentario y reinterpretación de la "Analítica": incluso si se dejan de lado los presupuestos ideológicos de sus interpretaciones, podría pensarse que la relativa indiferencia del crítico francés hacia el análisis del juicio de gusto supone ya una deformación del pensamiento kantiano. Pero una caracterización semejante sería igualmente culpable de exageración: es verdad que las exégesis de Lyotard no dan suficiente cuenta de la marginalidad de la "Analítica" y que en ese sentido pueden ser acusadas de unilateralidad, pero también es cierto que el "bloßer

Cfr. Kant 1990: \29, 119.

Cfr. Peña Aguado 1994: 50-51. 
Anhang" revela la plena fascinación de Kant por lo sublime, en la medida en que este, al exceder los límites de lo sensualmente representable, no supone un acuerdo de la imaginación con el entendimiento, sino con la propia razón. Kant coloca a la sublimidad por encima de lo bello, lo cual no deja de ser consistente con el papel subsidiario que aquella juega en la tercera crítica; por cuanto, al revelar la impotencia de la imaginación para comprehender lo desmesurado, incita al espectador a abandonar la captación del mundo exterior y a replegarse hacia la interioridad del espíritu, la sublimitas nos reenvía al pensamiento dualista, y a la discordia entre naturaleza y razón de la que Kant se encontraba convencido, y que solo se había intentado recubrir por medio del juicio de gusto. Esto remite a la función que Kant asigna a lo bello, consistente en establecer una reconciliación ilusoria entre la esfera del conocimiento y la de los principios morales; el hecho de que, según mostraremos, el juicio estético sublime represente una experiencia elitista en comparación con la que comporta el Geschmacksurteil, depende de la intolerancia de aquel frente a la ficticia reconciliación con lo natural propiciada por la belleza. Solo un "espíritu superior", educado en el estricto dominio de las inclinaciones instintivas, será capaz de tolerar una perfección estética que no se sustenta en la gratificación de los sentidos, sino en la subordinación del mundo material bajo el inteligible; el vulgo [Pöbel] (al que amedrenta la representación de lo informe) debe contentarse con la satisfacción inmediata que provee la contemplación de la belleza. Como en otras teorías (Bodmer, Breitinger, Pyra), también aquí conserva lo sublime una espiritualizada bigh seriousness por la que logra aventajar a la belleza; pero, entonces, debemos volver a preguntarnos cómo se justifican la brevedad y la relativa inconexión de la "Analítica".

Podría sugerirse que la postulación de un juicio estético apoyado sobre lo incorpóreo e informe, separado del sensus communis tanto como del anclaje en la realidad externa, emparentado con las leyes morales y ligado al desprecio de la naturaleza, podría representar una amenaza para la concreción artística y conducir, al mismo tiempo, a la exaltación de la ratio suprasensible. Observación quizás apropiada, pero que no parece haber guiado prioritariamente las elecciones kantianas; lo esencial es que la no mitigada franqueza de lo sublime, su reluctancia a ocultar la violencia ejercida sobre la naturaleza externa e interna y su entusiasmo, por el contrario, en celebrarla y exhibirla, hacen de ella una herramienta poco efectiva y capaz de concitar la oposición: la de los hombres "no ilustrados", que solo 
experimentan temor ante ella, pero también la de los demasiado sagaces, quienes advierten el peligro inherente al sacrificio de la realidad natural. Kant es consciente de que la sublimidad no es capaz de obtener adhesión inmediata: para gozar de ella, es necesario haber sido ganado previamente para la causa de la ley moral; por lo tanto, solo será razonable reservarla para instancias de excepción, y la convicción de que la intensidad de la sublimidad artística no admite un tratamiento sostenido - por lo que debe ser moderada con episodios atenuantes ${ }^{3}$ - es complementaria de la asociación de la polaridad bello/sublime con la oposición entre paz y guerra ${ }^{4}$. Si, por un lado, vemos que lo sublime es ofrecido como instrumento para prevenir el debilitamiento moral, también podemos concluir que - como la guerra - dicha experiencia constituye para Kant una instancia excepcional, una transitoria alternativa frente a la "demasiado pacífica" vida corriente. Tal vez sea más ajustado modificar los términos del problema, puesto que la distancia que separa a la guerra de la paz, y, por ende, la que media entre lo sublime y lo bello, no es en Kant tan considerable como podría pensarse de antemano; tal vez resulte más sensato analizar la paz, no ya como pura ausencia de tensiones, sino como una solución de compromiso en que las disensiones fundamentales y constantes del género humano (ante todo, aquella que divide a naturaleza y razón) han sido fugazmente acalladas. En Kant, la "pacífica" belleza es poco más que una ficción destinada a recubrir las contradicciones siempre vigentes; desde esta perspectiva, resultan insatisfactorios los periódicos intentos de subsumir a lo sublime bajo la rúbrica más general de la belleza ${ }^{5}$. No sería insensato postular lo contrario: si la paz no es otra cosa que una tregua provisional, un cese artificial de las hostilidades entre el reino de los fines y el de los imperativos morales, acaso debamos contemplarla como a una categoría subsidiaria, como una instancia mitigadora respecto de las menos frecuentes coyunturas en que se manifiesta la violencia desnuda. La paz es hija de la guerra, de un modo análogo a cómo la coerción engendra y engloba al consenso. Y en un sentido casi literal, puesto que el origen de los mecanismos consensuales se encuentra en la violencia. Así es que en el ocultamiento (pero no en la anulación) del

Cfr. Kant 1968: 829-830.

Cfr. Kant 1990: \28, 109

Así ocurre, por ejemplo, PeÑa Aguado 1994: 66-67. 
dominio de la naturaleza se encuentra el origen de lo bello; o, como indica Caygill: "To be at home in nature we first have to master it, to bring it under dominion, and then to efface our violence" (CAYGILL 1989: 347); correlativamente, lo sublime procede de una súbita revelación de las escamoteadas tensiones ("The sublime is the proportion of the beautiful without this effacement, the fear and trembling of violent appropriation", ibid.). Kant, que comprendía que el apócrifo funcionamiento consensual de la sociedad burguesa demanda el ocultamiento de la violencia primigenia, necesaria para la instauración de la ley, no ignoraba que esa misma violencia puede, bajo ciertas circunstancias, despertar la admiración o el respeto de los sojuzgados: es esta certeza la que lo conduce a atribuir al Jefe del Ejército mayor sublimidad que al hombre de Estado ${ }^{6}$. Lo sublime consiste, pues, en la revelación súbita de una enérgeia que en condiciones habituales se encuentra disimulada por el refinamiento y la urbanidad. Acaso en la asociación de lo sublime con la simplicidad (con la que Kant se integra a una larga tradición precedente) debamos encontrar la prueba más concluyente a favor del carácter de ilusoria distracción que posee la belleza ${ }^{7}$.

De momento, pasaremos por alto la mencionada afinidad entre la sublimitas y la segunda naturaleza suprasensible; nos interesa ahora detenernos en la tácita oposición entre simplicidad y "arte”. Así como lo sublime es simple y carente de artificio, la belleza consiste en una práctica del engaño a través de ornamenta, indispensables para que el sujeto perciba como verosímil el ficticio acuerdo entre naturaleza y racionalidad. Pero la sencillez estética de lo sublime (que, a su manera, encierra un artificio) no debe confundirse con la simplicitas de las leyes morales: la ausencia de adornos propia de la sublimidad es un analogon del imperativo moral. No sería inexacto decir que lo sublime no implica tanto la simplicidad misma cuanto la estetización de la simplicidad. En vista de lo anterior, podrá comprenderse el

Cfr. Kant 1990: \ 28, 108-109.

"Einfalt (kunstlose Zweckmäßigkeit) ist gleichsam der Stil der Natur im Erhabenen, und so auch der Sittlichkeit, welche eine zweite (übersinnliche) Natur ist, wovon wir nur die Gesetze kennen, ohne das übersinnliche Vermögen in uns selbst, was den Grund dieser Gesetzgebung enthält, durch Anschauen erreichen zu können” (KANT 1990: \29, 123). 
componente de verdad y el de distorsión inherentes a las dos posiciones que hemos enfrentado al comienzo; es decir: la de quienes minimizan el valor de la teoría kantiana de lo sublime dentro de la última crítica y la de aquellos que exaltan el valor de la sublimidad kantiana. En contra de lo propuesto por Peña Aguado, lo sublime extrae su mayor importancia del misterio y la ubicuidad: el papel aparentemente episódico, pero en verdad capital que juega la violencia en la dinámica habitual de la sociedad, es proporcional al exiguo pero trascendente espacio conferido a la "Analítica de lo sublime" dentro de la Kritike der Urteilskraft.

\section{Una estética de la abstracción: Kant, entre Shaftesbury y Burke}

Un táctica fructífera para empezar a dar cuenta de la significación histórica e ideológica de la teoría kantiana sobre lo sublime, es indicar las diferencias que la separan de algunas formulaciones que han ejercido influjo sobre ella. Un punto de referencia obligado es Shaftesbury: varias veces se han indicado las deudas que guarda la Kritike der Urteilskraft para con el autor de The Moralists (1711) en lo que se refiere a la reflexión sobre la sublimitas $^{8}$. La definición de lo sublime como lo que se encuentra ligado a la infinitud y, por tanto, situado fuera del campo de lo sensiblemente representable ha calado hondo en Kant ${ }^{9}$, como también lo ha hecho la descripción de esa misma experiencia como sentimiento dúplice, mezcla de encanto y temor ${ }^{10}$. Sin embargo, entre Shaftesbury y Kant existen discrepancias significativas: una de ellas, tal vez la esencial, es el modo desigual en que am-

8 El estudio más exhaustivo es el de Larthomas (1985: 303-332).

9 'Le 'beau', pour Shaftesbury, c'est l'expérience de la forme, de la 'limite' au sens platonicien. Le 'sublime', c'est l'expérience de l'infinité, de ce qui es apparemment en deçà, en réalité peut-être au delà de toute forme perceptible" (LARTHOMAS 1985: 304).

10 La ambigua actitud de Shaftesbury frente a la naturaleza sublime puede verse expuesta en The Moralists, ante todo en el detalle de los sentimientos que despierta la contemplación de las altas montañas. Así, dice Larthomas: "On ne peut pas ne pas constater cette réticence symptomatique de Shaftesbury devant le sublime, dont il éprouve pourtant si fortement le charme et la puissance. Il faut 
bos consideran a la naturaleza. Si bien persisten en Shaftesbury declaraciones de entusiasmo por la other-worldliness, el sentimiento de unidad con lo natural es lo bastante fuerte para evitar los gestos de desprecio con que Kant esporádicamente alude al reino de los fines. Para Shaftesbury (que en esto reanuda la fe en el principio de simpatía universal pregonado por el antiguo estoicismo) la naturaleza es un organismo regido por una voluntad inmanente, ante la cual el hombre debe irreflexivamente abandonarse. Un hombre provisto de alma no tiene que obedecer sino al universo; de ahí que el estremecimiento suministrado por lo sublime represente para Shaftesbury una prueba de la grandiosidad de la naturaleza, ante la cual el hombre se siente subyugado, y así lo testimonian las declaraciones de Theocles en The Moralists. Según Larthomas:

Le sublime est [pour Kant] l'occasion de découvrir en nous ce qui nous rend supérieurs à la nature. Cette idée est étrangère à Shaftesbury pour qui la raison, à la façon du logos stoïcien, n'est jamais que la loi interne de développement de la nature. Être supérieur à la nature n’a guère de sens dans une perspective moniste, comme celle qu'illustre Théocles: il s'agit bien plutôt de nous élever jusqu'à la plus haute puissance de la nature, en nous et hors de nous; c'est le propre du sublime que de nous faire sentir l'adéquation de notre force intérieure avec celle qu'anime, tout puissant, le Génie de la nature (LarTHOMAs 1985: 308-309).

Para esclarecer estas posiciones podríamos establecer un paralelo con un lector de Shaftesbury y un admirador entusiasta de la cosmovisión panteísta como lo es Goethe. Para este, la naturaleza violenta y amenazante, capaz de suscitar horror sagrado en los hombres, solo es maligna para quienes propulsan el sojuzgamiento de la realidad material; los lectores del primer

expliquer cette résistance. Le sublime présente quelque chose d'inquiétant qui, à première vue, met en déroute l'esthétique rassurante de la 'forme'. Si l'on s'arrête à l'exemple révélateur du grandiose de la haute montagne, les textes de Moralistes manifestent à cet égard une sorte d'ambivalence révélatrice. La montagne fascine, elle attire. Mais en même temps elle repousse, elle inquiète" (LARTHOMAs 1985: 306). 
Fausto recordarán que el espanto y el abatimiento fáusticos suceden a la confianza necia en la capacidad de sojuzgar al Erdgeist, este inmediato pasaje de la egolatría al arredramiento y el desánimo encuentra su contraparte en la vivencia de humilde pero eufórica unidad en el episodio "Wald und Höhle": solo en la espontánea renuncia a la voluntad de dominio radica la esperanza en una humanidad plena y pacificada con la naturaleza. Aquí, como, también sucedía en Shaftesbury, la visión de la naturaleza sublime no conduce a una instancia racional superadora, sino a la unión entre sujeto y objeto; la perfección de la especie no estaría, entonces, en la conversión del hombre en puro Vernunftwesen, sino en su más perfecta realización en cuanto Naturwesen.

No menos significativas son las disensiones respecto de Burke. El pensador irlandés ha ejercido una gravitación determinante durante el período antropológico de la estética kantiana, y su influjo aun se deja sentir en la tercera crítica; en la asociación de lo sublime con lo inhabitual y extraordinario, con las determinaciones de los poderes tiránicos y, en particular, con la supremacía masculina, se advierte la influencia de la Pbilosophical Inquiry. Kant ha aprendido de Burke que la naturaleza solo es sublime donde se muestra reluctante a los deseos de posesión, donde rehusa someterse a la expoliación humana. Cuando en la Kritike der Urteilskraft se afirma que el juicio estético puro de sublimidad es incompatible con aquellos animales que poseen un fin determinado $^{11}$, y que solo ha de hallarse en la naturaleza indómita, se perciben ecos de la convicción burkeana de que los animales sublimes son aquellos que no se dejan supeditar a la utilidad del hombre:

11 Esta tesis parecería contradecir nuestras ulteriores consideraciones en torno a la justificación kantiana de la represión de la naturaleza. Pero cabe recordar que, luego de afirmar la autonomía de la naturaleza indómita, Kant confirma, la inferioridad de esta frente a la grandeur de la ratio. Ya Adorno ha puesto de relieve que el aparente reconocimiento de la autonomía de la naturaleza presente en la teoría kantiana de lo sublime está puesto, en realidad, al servicio de una justificación del bárbaro dominio ejercido por el hombre: "Indem er [= Kant] jedoch das Erhabene ins überwältigend Große, die Antithese von Macht und Ohnmacht setzte, hat er ungebrochen seine fraglose Komplizität mit Herrschaft bejäht. Ihrer muß Kunst sich schämen, und das Nachhaltige, welches die Idee des Erhabenen wollte, umkehren” (Adorno, 1998: 7, 296). 
The horse, in the light of a useful beast, fit for the plough, the road, the draft; in every social, useful light, the horse has nothing sublime; but it is thus that we are affected with him, whose neck is clothed with thunder, the glory of whose nostrils is terrible, who swalloweth the ground with fierceness and rage, neither believeth that it is the sound of the trumpet? In this description, the useful character of the horse entirely disappears, and the terrible and sublime blaze out together (BURKE 1963: 56).

Pero son sustanciales las diferencias que separan al "uso" kantiano de la categoría de sublimidad de la interpretación burkeana. En la "Analítica" se destacan las desavenencias entre el tratamiento trascendental del juicio estético y el análisis psicológico desarrollado por Burke: al basarse en criterios empíricos, las conclusiones de la Philosophical Inquiry, como las de toda estética empirista, se circunscriben al nivel de los Privaturteile (propios de lo agradable), sin que sea posible para ellas elevarse hasta la universalidad; aquí, afirma Kant, desaparece toda crítica del gusto. Si, por el contrario, postulamos el derecho de exigir el asentimiento universal, tenemos que dejar que nuestros juicios se basen en un principio a priori, hasta el cual es imposible elevarse partiendo de la investigación de las normas empíricas. Esto nos remite al hiato entre realidad empírica y trascendente (entre lo singular concreto y la universalidad abstracta, entre las contradicciones propias de la "tangible" realidad burguesa y la elevación abstracta de su proyección ideal) del que Kant no ha podido librarse, en parte por la carencia de bases históricas y sociales explícitas y conscientes en su pensamiento.

Kant veía con simpatía los presupuestos subjetivistas de Burke, la insistencia de este en analizar la sublimidad y la belleza, no a partir de las cualidades intrínsecas del objeto - los escenarios naturales, las obras artísticas -, sino del estudio de las reacciones en el sujeto receptor. Pero Kant rechaza la insistencia del escritor irlandés en estudiar las repercusiones físicas, corporales de la experiencia estética ${ }^{12}$. Y es que en Burke, según apunta

12 Recordemos que, en Burke, lo sublime no está colocado solo al servicio de la formación de un gran alma, sino también de un "cuerpo fuerte"; cfr. PEÑA AguAdo 1994: 34-35. Es revelador que Kant justifique la universalidad del juicio 
Eagleton, no solo subsiste la pretensión fundacional de la estética consistente en establecer - desde Baumgarten - una "política del cuerpo"; también cobra vida el ideal antiilustrado consistente en calcar la dinámica de las relaciones sociales sobre el modelo de la articulación orgánica del individuo $^{13}$. El análisis de Eagleton basta para mostrar que los planteos estéticos burkeanos suponen una dinámica historicista que en vano trataríamos de rastrear en el pensamiento de Kant; pero no solo eso: también un reconocimiento de los límites de la legislación abstracta promovida por el racionalismo burgués. Podrá recalcarse el carácter reaccionario del tradicionalismo burkeano, pero algunas de sus críticas a la legislación racionalista captan con lucidez el animus de la sociedad burguesa. La insistencia de este defensor del ancien régime sobre el valor de la experiencia compartida, de las manners y los sobreentendidos ideológicos sobre los que halla asentada toda cultura, cuadra, por lo demás, con su interés y destreza para la oratoria, aquella disciplina que, emparentada con la doxa, desde antiguo, ha tomado bajo su cargo la tarea de colocar lo no dicho al servicio del adoctrinamiento y la exaltación de los ánimos. Es sabido que la filosofía racionalista - aliada, en este sentido, del espiritualismo - se ha constituido en enemiga de la retórica, al negar validez a la opinio y al elevar a las ideas claras y distintas al rango de paradigma de todo conocimiento. Lo indistinto, tanto como lo verosímil, representaban, para el antirretoricismo cartesiano, indicios de error; y huellas de estos prejuicios perduran en el escepticismo kantiano frente a la elocuencia. Burke, en contra de tales tendencias, anteponía " [...] the conative over the cognative, rhetoric over representation" (EAGLETON 1995: 51); sabía que los valores morales, éticos, políticos o religiosos, solo pueden implantarse extrínsecamente sobre una comunidad bajo el riesgo de destruir sus valores esenciales. Más allá de su conservadurismo político, el autor de las Reflections on the Revolution in France (1790) supo percatarse de los riesgos de una disolución absoluta de los valores tradicionales, como también del peligro de imponer valores trascendentes sobre una realidad

reflexionante estético en la general semejanza de nuestras Erkenntnisvorbedingungen, en tanto Burke justificaba la identidad de nuestras evaluaciones estéticas en la uniformidad de los órganos sensitivos.

13 Cfr. Eagleton 1995: 51. 
de hecho. Detrás de las críticas a la legislación burguesa podemos presentir la sospecha de que la abstracción burguesa no puede resultar sino ineficaz ante la infinidad de componentes emotivos, siempre intuidos pero jamás explicitados, que constituyen el material mismo de la ideología. Estos restos no asimilados por el pensamiento sistemático coinciden con las cualidades que Lionel Trilling ha reunido en su célebre definición de las manners:

Somewhere below all the explicit statements that a people makes through its art, religion, architecture, legislation, there is a dim mental region of intention of which it is very difficult to become aware. We now and then get a strong sense of its presence in the past but by reason of its absence. As we read the great formulated monuments of the past. We notice that we are reading them without the accompaniment of something that always goes along with the formulated monuments of the present [...] is a culture's hum and buzz of implication. I mean the whole evanescent context in which its explicit statements are made. It is that part of a culture which is made up of half-uttered or unuttered or unutterable expressions of value (Trilling 1961: 231-232).

En relación con esto deberíamos considerar algunos puntos de la crítica de Burke a la sociedad burguesa, de los que puede deducirse un contraste con Kant: por un lado, el pensador irlandés advierte, proféticamente, la vinculación entre el avance del individualismo burgués y la disolución de las particularidades específicas propias de las comunidades humanas; entre la desaparición de lo que Raymond Williams ha denominado knowledgeable community, y el nacimiento de la concepción política, jurídica y filosófica del individuo como ser abstracto y aislado, existen múltiples afinidades; de acuerdo con Burke, el individuo solo puede realizarse en el seno de la comunidad, pero no de una comunidad humana abstracta, sino de aquella a la que oriundamente pertenece. Williams destaca que, para Burke: "The whole progress of man is thus dependent, not only on the historical community in an abstract sense, but on the nature of the particular community into which he has been born. No man can abstract himself from this; nor is it his alone to change" (Wildiams 1960: 9). En segundo 
lugar, Burke estima que los efectos del racionalismo moderno - y, ante todo, la desarticulación del pensamiento y la actividad humanas en esferas autónomas - son resultado de la disolución de los valores comunitarios y tradicionales, de la desaparición de aquello que él denominaba spirit of the nation. La verdadera sublimidad es la que, en contradicción con el convencionalismo y la legislación abstracta de la civilización burguesa, se encuentra sustentada en los ritmos periódicos y ancestrales, las leyes no escritas pero consagradas por el tiempo y tácitamente obedecidas. Burke sentía horror ante las súbitas soluciones de continuidad:

He speaks from the relative stability of the eighteenth century against the first signs of the flux and confusion of the nineteenth century, but he seeks also against those rising doctrines which the eighteenth century had produced, and which were to become the characteristic philosophy of the change itself [...] He established the idea of the State as the necessary agent of human perfection, and in terms of this idea the aggressive individualism of the nineteenth century was bound to be condemned. He established, further, the idea of what has been called an 'organic society', where the emphasis is on the interrelation and continuity of human activities, rather than on separation into spheres of interest, each governed by its own laws (Williams 1960: 11).

Desde esta perspectiva, la ética y la estética burkeanas se encuentran en las antípodas de una filosofía como la de Kant, la cual no solo ha nacido de la crisis de valores que Burke describe y condena, sino que ha llegado a constituir uno de sus más firmes fundamentaciones. De ahí que, si la violenta energía de la sublimitas reside, de acuerdo con el autor de la Philosophical Inquiry, en la comunidad tradicional, para el filósofo alemán ella se encuentre anidada en esa ratio autónoma que ha hecho posible la destrucción de las ancestrales Gemeinschaften.

La indiferencia de la teoría artística kantiana hacia las condiciones concretas de la vida comunitaria es tan llamativa como la desatención por la experiencia sensible; es revelador que la estética, que nace en Baumgarten como disciplina destinada a otorgar derecho de ciudadanía a la sensibilidad, conduzca en Kant a la represión y, a la vez, la sublimación de lo sensitivo. 
El ánimo de concentrar la reflexión en los principios a priori antes que en la dimensión empírica de la naturaleza y del arte, constituye la contrapartida de la aspiración primigenia de reconocer relevancia a la realidad concreta y tangible. El hecho de que Kant definiese a los productos de la imaginación como "ideas estéticas" -y no como conceptos estéticos- es significativo. Frank Lentricchia ha indicado que de aquí puede deducirse tanto la depreciación de la experiencia sensitiva cuanto la circunscripción de los juicios estéticos al campo de lo puramente ficcional:

When he [Kant] terms the representation of imagination, in its aesthetic phase, an 'aesthetic idea', he means for us to recall the key distinction of ideas and concepts. The latter as 'forms', because they find adequate 'content' in the sensuous manifold, yield the cognitive shapes of experience; the former, as forms of pure reason ('rational ideas') which cannot be satisfied in time, and cannot be adequately filled out with the sensuous data of 'intuition', point beyond the limits of experience and are, from these perspectives, 'fictions' in the sense that they produce no cognition of the phenomenal world. In the cognitive privilege given to the 'concept' over the 'idea' we see the surreptitious back-door entry of ontological realism into Kant's system: the concept gives knowledge because, as a realist would insist, its formation begins in, and is respectful of, a passive reception of the manifold (LeNTRICCHIA 1980: 40).

El conocimiento - más allá de las propensión de la epistemología kantiana al idealismo subjetivo - requiere de la presencia de una percepción, de una "cierta afección del espíritu" mediada por la sensibilidad. Las ideas, desde su situación de elevada trascendencia, no pueden condescender a adecuar sus determinaciones a priori al material ofrecido por la sensibilidad, y esto vale tanto para las ideas racionales como para las estéticas, aunque de variada forma:

The 'rational idea' (God, freedom, immortality) [...] is empty because it is many sizes too big for the sensory data it would envelop; and the 'aesthetic idea' is epistemologically blind because as an unconstrained sensory synthesis, an exuberant revelling in the senses, it bursts through the conceptual frame which would contain it and give it phenomenal focus (LENTRICCHIA 1980: 40-41). 
La idea estética dispone, pues, de los datos ofrecidos por la sensibilidad sin mediación de conceptos, y en esa medida se encuentra libre de toda determinación natural; concebida como un libre juego y liberada del peso de la realidad fenoménica, dicha idea coadyuva a instituir una estética para la cual la experiencia artística representa una pura ficción, una realidad vicaria generada con fines evasivos. De ahí la paradoja de la estética kantiana, que al tratar de ensalzar la vivencia artística definiéndola como ficción pura y sustrayéndola al contacto con la realidad fenoménica, concluye degradándola al nivel de un divertimento:

In telling us that art does not mold, but rather remolds, Kant trivializes what those in his tradition would empower against a culture all too ready to denigrate fictions as a holiday from our 'serious' transactions with the world. His intention of isolating the distinctive character of the aesthetic experience was admirable, but his analysis resulted in mere isolation. By barring that experience from the truth of the phenomenal world, while allowing art's fictional world entertainment value, he became the philosophical father of an enervating aestheticism which ultimately subverts what it would celebrate [...] The place 'outside' of art encloses, defines and judges fictions as pleasant lies generated out of boredom, fantasies which briefly block out the phenomenal reality to which we all, as serious, rational persons, shall return when we regain our courage (LENTRICCHIA 1980: 41-42).

Pero si el juicio de gusto, dada su ubicación intermedia entre conocimiento y moralidad, preserva un débil contacto con la naturaleza, el juicio estético sublime representa un progreso hacia la esfera de las ideas racionales. Teniendo en consideración que el lazo de lo sublime con la naturaleza externa es menor en el caso de la sublimidad que en el de lo bello, no sería inexacto decir que en ella se expresa desdén hacia el ámbito del conocimiento empírico.

\section{Sublimidad y ascetismo: Kant y la represión de la naturaleza}

En la Kritik der Urteilskraft, la oposición entre lo bello y lo sublime carece de dimensión histórica: en esto, Kant diverge de otros teóricos precedentes de la sublimidad: en la "Analítica de lo sublime", como en la de lo 
bello, dichas categorías se presentan como "enraizadas en la naturaleza humana" y por ende se las considera - tal como al juicio reflexionante estético en general - en cuanto capacidades intrínsecas del hombre, componentes indefectibles de la conditio bumana. La adopción de una óptica trascendente condiciona la ahistoricidad del modelo, lo que difícilmente hubiese podido sostenerse de haberse adoptado una perspectiva más próxima al empirismo. Con raras excepciones, Kant no parecía dispuesto a conceder que, en materia artística, la verdad se encuentra sujeta a determinaciones históricas; por tanto, ni la belleza ni la sublimidad se hallan en su exposición emparentadas con una época o una nación en especial, sino que constituyen un patrimonio de la humanidad latissimo sensu. Sin embargo, en el formalismo estético kantiano, en la postergación de la sensual materialidad, en la preferencia (en el caso de lo sublime) por un arte no representativo y apartado de lo mimético, es imposible no advertir las huellas de su ambiente y de su tiempo; y en tal medida que esta teoría, no a pesar de sus pretensiones abstractas y universalistas, sino justamente a causa de ellas, acaba por ser la más conspicua encarnación de la sublimidad germánica. La desconfianza del ascético Kant ante la naturaleza externa e interna resulta una coronación de los empeños espiritualistas de los suizos y Pyra o - en menor medida - de Moses Mendelssohn: lo cual no solo se observa en la concepción de lo sublime, sino aun en la austera visión de la belleza desplegada en la tercera crítica. Cuando Eagleton afirma que la ley moral, a pesar de su radical antiesteticismo, no hace más que calcar las cualidades formales de lo estético, solo comprende parcialmente el problema ${ }^{14}$; lo que se observa en Kant es más elemental: una polarización entre lo trascendental y lo empírico que implica al conocimiento teórico, al juicio estético y a la praxis moral, y que justifica las mutuas homologías. No sería errado afirmar que cada campo encierra una estructura análoga a la de los restantes.

Parece ya superfluo indicar que, en Kant, la forma en que lo sublime aventaja a lo bello es análoga a aquella en que, en términos más amplios, lo racional supera a lo empírico, o (para remitinos a la raíz de estas cuestiones) en que el etéreo citoyen flota libremente por encima del tan real como

14 Cfr. Eagleton 1991: 81. 
prosaico bourgeois. Alasdair MAcInTYRe (1981: 22 y ss.) y EAGLETON (1991: 80-81) han destacado que el formalismo ético kantiano es resultado de una situación histórica en que las cuestiones morales han dejado de ser comprendidas a partir de un background de relaciones sociales establecidas; en buena parte de los sistemas morales precedentes y, principalmente, en la tradición iniciada por la ética aristotélica, las máximas morales eran deducidas de la observación de la vita activa; la determinación del recto proceder suponía un conocimiento de la estructura social sobre la cual que debían aplicarse las reglas concretas. Es en el pensamiento kantiano donde se consuma la autonomización de las máximas morales respecto de las condiciones concretas de la vida social:

In certain forms of pre-bourgeois society, the question of how a subject ought to behave is closely bound up with its location within the social structure, so that a sociological description of the complex relations in which an individual stands would inescapably involve a normative discourse too. Certain rights, duties and obligations are internal to social functions, so that no firm distinction is possible between a sociological idiom of fact and an ethical discourse of value. Once the bourgeois social order begins to reify fact, and to construct a kind of human subject transcendentally prior to its social relations, this historically grounded ethics is bound to enter into crisis. What one ought to do can no longer be deduced from what, socially speaking, one actually is. [...] If one can return no social answer to the question of how one ought to behave, then virtuous behaviour, for some theorists at least, must become an end in itself. Sollen is removed from the sphere of historical action and analysis; one must behave in a particular way simply because one must (EAGLETON 1991: 80-81).

Este intento de reformar la vida práctica partiendo del imperativo ético no deja de evocar la situación política y social de Alemania, pero no lo hace menos el hecho de que Kant considere esa sumisión de la realidad "de hecho" bajo los valores trascendentes como una derivación de la naturaleza del hombre, y que por tanto asigne las derivaciones de pensamiento moral a la humanidad in toto, sin reparar en las diferencias concretas entre épocas y civilizaciones. Acierta, pues, el joven Hegel cuando observa en este 
formalismo el indicio seguro de la desintegración social: la conocida aseveración hegeliana de que la necesidad de unificación por medio de leyes surge solo cuando la vis viva de una sociedad ha perdido su originaria capacidad de cohesión, representa una justificada crítica a la hipostatización kantiana del Sollen. El concepto de positividad, acuñado por Hegel para diferenciar al cristianismo de la religiosidad pagana, constituye una denuncia indirecta del formalismo de la ética de Kant y de Fichte ${ }^{15}$. Para estos, el espacio de la actividad humana se restringe a la aceptación de los preceptos abstractos; se trataba de adecuar a la sociedad burguesa existente con su representación ideal, "sublimada", la cual carece de antagonismos internos:

Für Kant und Fichte ist in der Moral diese Trennung [entre lo sensible y lo espiritual] zugleich ein Ausdruck und ein philosophisches Mittel dazu, um ihre Kritik der Moral des Menschen ihrer Zeit mit einer Bejahung der bürgerlichen Gesellschaft zu vereinigen. In der rein geistigen Sphäre des 'kategorischen Imperativs' konstruiert dann Kant und nach ihm Fichte ein Idealbild der bürgerlichen Gesellschaft, in welchem die bedingungslose Hingebung an die überirdische, geistige, nicht mehr der Welt der Phänomene angehörende 'Pflicht' konfliktlos und harmonisch funktioniert. Alle Gegensätze und Widersprüche in der bürgerlichen Gesellschaft der Wirklichkeit reduzieren sich nun auf den Gegensatz des sinnlichen und des moralischen Menschen, des 'homo phänomenon' und ‘homo noumenon'. Wenn die Menschen ganz dem Sittengesetz entsprechend leben würden, würde es in der bürgerlichen Gesellschaft keinerlei Konflikte oder Widersprüche geben. [...] In diesen Auffassungen drücken sich zwei gesellschaftlich wichtige Anschauungen aus. Erstens die Moralität der ersten, der asketischen Periode der bürgerlichen Entwicklung, die radikale Spiritualisierung und das in den idealistischen Himmel

15 En Der junge Hegel, LukÁcs señala que, para el autor de la Phänomenologie, la positividad es la realidad social que corresponde al dualismo ético kantiano. Por tanto, el carácter dado, muerto y objetivo de la realidad histórica y social no es, de acuerdo con los análisis hegelianos, el resultado de una posible "naturaleza humana", sino el producto de una evolución histórica contingente y, por tanto, modificable. 


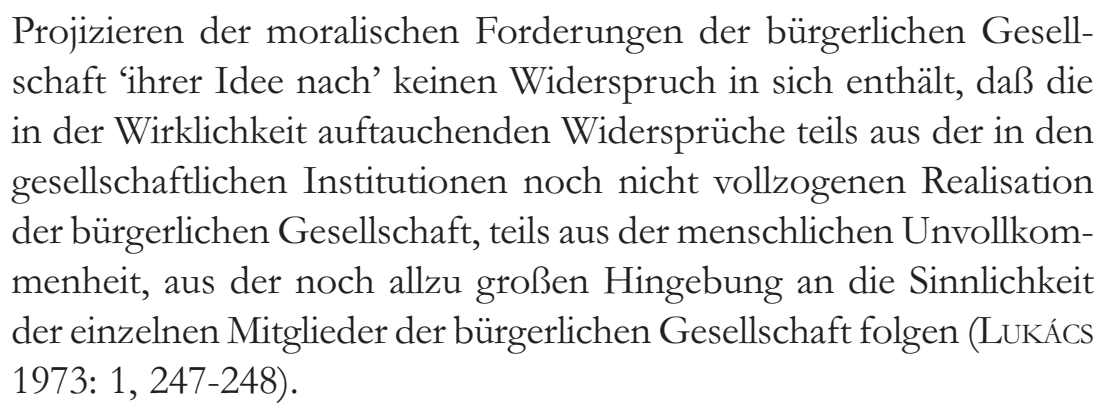

En esa espiritualización radical podríamos ver un componente del ascetismo burgués imperante tanto en la esfera de la economía como en la de las mores: nos referimos al principio que determina la represión del placer inmediato (ligado a la gratificación corporal) y su reemplazo por el placer retardado resultante del acallamiento de la sensibilidad -. El hecho de que Kant concibiese al bien moral como una imposición antiestética y contrapuesta a la satisfacción de los sentidos, aunque capaz de producir el bien general en forma mediata, autoriza una lectura de la segunda crítica en términos de la metapsicología freudiana, como una justificación de la victoria del principio de la realidad sobre el intuitivo e inmediatamente gratificante principio del placer ${ }^{16}$; de ser así, nada impediría aplicar a la ética kantiana las objeciones dirigidas por Horkheimer y Marcuse contra las ideologías empeñadas en defender la renuncia a la vida instintiva en beneficio de las exigencias de la sociedad burguesa: afirmados en una relectura de Freud, ambos pensadores han denunciado que, entre las cualidades más características de la sociedad burguesa, se encuentra la liquidación o, al menos, la drástica reducción de los placeres inmediatos, que deben ser sacrificados con el fin de contribuir a la preservación del totum social ${ }^{17}$. La moral del deber ser, que exige la inmolación de los deseos personales a manos del todo, acompaña y consu-

16 De hecho, EAGLETON se encarga, aunque lateral y parcialmente, de considerar las antinomias de la ética kantiana desde esta perspectiva.

17 "Was sich in der Philosophie als Verpönung von Triebregungen ausdrückt, erweist sich im wirklichen Leben als die Praxis ihrer Unterdrückung. Alle Instinkte, die sich nicht in vorgezeichneten Bahnen bewegten, jedes unbedingte Glücksverlangen wurde zugunsten 'sittlichen', auf das 'Gemeinwohl' bezogenen Strebens verfolgt und zurückgedrängt; und im gleichen Maß, wie dieses Gemeinwohl den unmittelbarsten Interessen der meisten widersprach, entzog sich der Übergang 
ma la opresión de la naturaleza externa; para la cosificadora ideología burguesa, el goce individual debe ser eliminado, tal como ocurre con toda particularidad que no se adecua a la dinámica de lo universal. Las prédicas idealistas a favor de la abnegación y la liquidación de las pasiones pretenden anular la capacidad del individuo para el placer inmediato ${ }^{18}$. Estos elementos están presentes en la moral de Kant; solo que deberíamos tener en cuenta un detalle suplementario, y es que el espiritualismo kantiano no se explica solo como derivación del ascetismo capitalista, sino también por la presencia de un componente preburgués, vinculado con las particulares condiciones de la evolución germánica. La aseveración marxiana según la cual Lutero ha conseguido introducir el fraile en el interior del corazón humano, podría aplicarse al pensamiento ético kantiano. No nos corresponde especificar los puntos en común entre el ascetismo protestante y el burgués; solo nos interesa señalar que en Kant confluyen elementos provenientes de las dos ideologías y que de ello depende la disposición a introyectar la servidumbre en la consciencia de los dominados. Esta cualidad de la ética de Kant fue advertida ya por Hegel, quien en Der Geist des Christentums und sein Schicksal (1798-1800) advierte que la ética autónoma no supera la positividad de la religión cristiana, sino que la traslada al interior del sujeto:

psychischer Energien in sozial erlaubte Formen rationaler Begründung, bedurfte die Gesellschaft zur Domestizierung der Massen neben dem materiellen Zwang einer durch Religion und Metaphysik beherrschten Erziehung [...] Selbstdisziplin und Verträglichkeit untereinander und gegenüber den Herrschenden wurden ihnen durch alle Mittel der Gewalt und Überredung beigebracht. Die Individuen wurden gebändigt" (HoRKHEIMER 1970: 103).

18 "Der Mensch, wie er sein soll, das Musterbild, das der bürgerlichen Anthropologie überall zugrunde liegt, hat ein bedingtes Verhältnis zum Genuß, es ist auf 'höhere Werte' ausgerichtet. Im Leben der vorbildlichen Menschen nimmt die Lust, in ihrer unmittelbarsten Form als geschlechtliche und weiterhin als materielle Lust überhaupt, einen geringen Platz ein. Die Arbeit, die das Individuum für sich und andere verrichtet, geschieht um hoher Ideen willen, die mit der Lust, wenn überhaupt, nur ganz lose zusammenhängen [...] Die Fähigkeit zu unmittelbarer Lust ist vielmehr durch die idealistische Predigt der Veredelung und Selbstverleugnung geschwächt, vergröbert, in vielen Fällen ganz verloren [...]" (HorkHEIMER 1970: 104-105). 


\begin{abstract}
Durch diesen Gang ist aber die Positivität nur zum Teil weggenommen; und zwischen dem tungusischen Schamanen mit dem Kirche und Staat regierenden europäischen Prälaten oder dem Mogulitzen mit dem Puritaner und dem seinen Pflichtgebot Gehorchenden ist nicht der Unterschied, daß jene sich zu Knechten machten, dieser frei wäre; sondern daß jener den Herrn außer sich, dieser aber den Herrn in sich trägt, zugleich aber sein eigener Knecht ist; für das Besondere, Triebe, Neigungen, pathologische Liebe, Sinnlichkeit, oder wie man es nennt, ist das Allgemeine notwendig und ewig ein Fremdes, ein Objektives; es bleibt eine unzerstörbare Positivität übrig, die vollends dadurch empörend wird, daß der Inhalt, den das allgemeine Pflichtgebot erhält, eine bestimmte Pflicht, den Widerspruch eingeschränkt und allgemein zugleich zu sein enthält und um der Form der Allgemeinheit willen für ihre Einseitigkeit die härtesten Prätentionen macht. Wehe den menschlichen Beziehungen, die nicht gerade im Begriff der Pflicht sich finden, der, sowie er nicht bloß der leere Gedanke der Allgemeinheit ist, sondern in einer Handlung sich darstellen soll, alle anderen Beziehungen ausschließt oder beherrscht (HEgEL 1971: I, 323).
\end{abstract}

La caracterización de la ética kantiana como estrategia de desplazamiento de la tiranía al interior de la consciencia moral, ayuda a comprender cuánto más sutil, pero también cuánto más efectivo es el funcionamiento del subjetivismo moral frente a otras formas más evidentes y, por tanto, más abiertamente cuestionables de despotismo. Más allá de sus pretensiones universalistas, el imperativo categórico recarga sobre la sensibilidad corpórea del individuo un yugo comparable al que la sociedad burguesa impone sobre los deseos sensoriales de sus sometidos: si en la vida corriente los hombres son empíricamente obligados a sacrificar su bienestar inmediato para cumplir, a largo plazo, con los designios del conjunto, ¿qué otra ideología podría ser más adecuada que aquella que busca convencerlos de que ese sacrificio no es contingente, sino que ha sido prescripto por las leyes trascendentales de la más alta razón? Y si el individuo es inducido a inmolar su gratificación física en función de la idea, todo gesto de rebeldía, toda ocasional negativa a cooperar con el sistema vigente es presentado como un atentado contra el orden trascendental, como un delito contra las determinaciones de la razón. Pero si la grandeza moral exige que el logos se libere de la materia - que el sujeto sojuzgue y, por ende, consiga enajenarse 
de la naturaleza externa e interna -, esa perfección última solo podrá ser simulada por aquellos que a través de su efectiva situación de dominio han conseguido distanciarse de las actividades materiales.

La tiranía de la ley abstracta, tal como han mostrado Horkheimer y Adorno en Dialektik der Aufklärung (1944), es la objetivación filosófica del dominio económico real: era necesario que el amo colocase al servidor entre sí mismo y la materia para que se consumase la emancipación del logos señorial y la sumisión del mundo externo. La preservación del orden social coloca al amo en la situación de sacrificar la existencia material de sus súbditos, pero también obliga a estos a introyectar las determinaciones del señor en la intimidad de los propios espíritus: la consciencia moral actúa, pues, a la manera de un déspota que ordena la obliteración de la satisfacción corpórea inmediata para colaborar en el sostenimiento del todo ${ }^{19}$. Recíprocamente, el amo debe incorporar dentro de sí mismo al despreciado siervo: lo hace rebajando sus facultades instintivas a la altura de una opresiva servidumbre, acallándolas y ordenándoles rendir la debida subordinación a la ratio. El ascetismo burgués se exterioriza tanto en la exigencia del capitalista de acallar sus deseos de satisfacción inmediata a fin de perpetuar la acumulación, como en la obligación, constrictivamente impuesta a las clases inferiores, de colocar el propio cuerpo al servicio de una voluntad ajena. Desde esa perspectiva, es posible decir que amos y servidores coinciden - aunque de distintas formas y con secuelas diversas - en el sacrificio de lo instintivo a manos de la ratio ${ }^{20}$, pero existe entre ambos grupos la diferencia de que solo los señores, en la medida en que han colocado al siervo

19 Marcuse ha explicado de qué modo la introyección en el interior de la psique de la represión externa conducida por los amos está puesta al servicio de la preservación de las instituciones vigentes: "The struggle against freedom reproduces itself in the psyche of man, as the self-repression of the repressed individual, and his self-repression in turn sustains his masters and their institutions. It is this mental dynamic which Freud unfolds as the dynamic of civilization" (MARCUSE 1973: 32).

20 Horkheimer y Adorno han explicitado esta doble expresión del ascetismo - el de los dominadores y el de los dominados - al analizar el carácter "protoburgués" de Odiseo" y la condición proletaria avant la lettre de los navegantes bajo su mando; cfr. AdORNo 1998: III, 52. 
entre ellos y la materia, pueden proyectar la imagen de una identificación plena con la idea y observar a los sometidos con la misma superioridad con que el espíritu trasciende a la existencia corpórea. Si el desarrollo de la civilización burguesa no ha sido solo el correlato del avance de la división del trabajo, sino también del progreso de la represión de los deseos sensoriales, todo ello ha ocurrido en aras de exaltar el valor de la producción por sobre el del consumo: aun cuando el capitalismo ha creado una descomunal industria consumista, para la cual no es posible encontrar parangón en el pasado, la ideología propulsada por la burguesía exige el acallamiento de la sensibilidad (de la misma manera que la desmesurada plétora de bienes materiales ha podido conceder hegemonía a las ideologías espiritualistas). Es por todo esto que Eagleton identifica a las categorías de lo bello y lo sublime, respectivamente, con los conceptos de consumo y producción:

Like Kant's aesthetic object, the commodity would seem designed especially for our faculties, addressed to us in its very being. Viewed from the standpoint of consumption, the world is uniquely ours, shaped to nestle in our palms; seen from the standpoint of production it appears, like Kantian Nature, as an impersonal domain of casual processes and autonomous laws [...] One can trace something of this movement in the dialectic of the beautiful and the sublime. If things-in-themselves are beyond the reach of the subject, the beautiful will rectify this alienation by presenting reality, for a precious moment, as given spontaneously for that subject's powers. If this then seems likely to breed complacency, the sublime is always on hand with its intimidatory power; but the dangerously demoralizing effects of such power are in turn tempered by the subject's joyful consciousness that the power in question is that of its own majestic Reason (EAGLETON 1991: 174-175).

Para comprender las repercusiones de esta problemática sobre la estética de Kant, podríamos remitirnos a un paralelo que establece Lyotard en forma provisoria, pero ante cuyas consecuencias últimas retrocede: la equiparación del dispositivo sacrificial que conlleva la aparición de lo sublime con el despliegue de la dialéctica entre amo y esclavo; el cálculo de intereses por el que "La nature est sacrifiée sur l'autel de la loi" (LYOTARD 1988: 174-175), es parangonado con aquel por el cual el esclavo es persua- 
dido de la necesidad de resignar su independencia material para obtener un reconocimiento en la esfera moral:

L'usage contingent' de la nature procède donc d'une économie sacrificielle des pouvoirs facultaires. L'égard que le sublime adresse à la loi s'obtient, et se signale, par un usage des formes qui n'est pas celui auquel elles destinent et sont destinées d'elles-mêmes [...]. Comme dans tout dispositif sacrificiel, il y a là un calcul des intérêts, un escompte sur les sentiments. Résilie la faveur, tu auras l'égard. Il parait facile de recouper ce calcul avec celui qui soutient une dialectique (par exemple, maitre-esclave: renonce à la jouissance, tu auras la reconnaissance) (LYOTARD 1988: 174-175).

Lyotard pretende mitigar el ultraje afirmando - à la Girard - que la inmolación de la realidad material tiene por efecto la sacralización de la naturaleza: es difícil imaginar de qué modo se realiza la reparación por la afrenta, sobre todo en vista de que el crítico francés prescinde de toda explicación y se limita a enunciar lateralmente la idea:

Mets le feu au beau pour que, de ses cendres, le bien te revienne. Tout sacrifice comporte ce sacrilège. Le pardon ne s'obtient que par l'abandon, la mise au ban, d'un don premier, qui doit lui-même être infiniment précieux. La nature sacrifiée est sacrée. L'intérêt sublime évoque un tel sacrilège. On est tenté de dire: un sacrilège ontologique (LYOTARD 1988: 174).

Esa promesa de redención de la naturaleza es tan irrealizable como los compromisos del amo para con sus siervos; o - para retomar las reflexiones de Horkheimer y Marcuse - tan vana como las recompensas de felicidad futura que los poderes establecidos certifican para justificar los sacrificios del individuo. La ley es tan indiferente al placer como al displacer: procede con total desinterés ante las necesidades y ante la existencia de los sujetos empíricos ${ }^{21}$; por tanto, las determinaciones de la razón solo reclaman ciego

21 Hecho que el propio LyOTARD reconoce: "La loi ne te veut pas de mal, elle ne te veut rien" (1991: 168). 
respeto, y no admiten hacer concesiones al deseo de los sujetos empíricos. Para seducir a estos, la ley necesitaría corporeizarse, abandonar su apariencia inmaterial y convertirse en un phaenomenon - menos en el sentido kantiano que en el que Baumgarten concedía al término, es decir: transformarse en apariencia visible, presentación concreta -. Eso contradiría la naturaleza íntima de la ley, que promete dignidad moral a quienes actúan en contra de sus impulsos afectivos, a quienes renuncian a la felicidad material y abrazan la Idea. Al desdeñar las exigencias de la carne, el hombre virtuoso testimonia su respeto por la causalidad absoluta; y, sin embargo, aunque sustraído a la allure de la mera belleza, el irrepresentable bien moral es susceptible de ser representado; o - mejor aun - es viable que la imaginación "presente" la incongruencia de las ideas racionales con nuestra facultad de representación, y que eso redunde en una experiencia placentera (en un "placer negativo"). Y la exteriorización de ese goce arrebatador pero carente de voluptuosidad es el entusiasmo, al que Kant enlaza con la sublimitas: si esta afección no puede identificarse ni contender con la neutra Achtung, puede en cambio colocarse al servicio de la potencia más alta, excitando en los sujetos empíricos la admiración por las inmateriales e "irrepresentables" leyes de la razón. En otros términos: el reconocimiento de una facultad, contenida en nuestro espíritu, para rechazar lo inmediatamente agradable y abrazar un bien que repugna a la sensibilidad, excita nuestro entusiasmo y revela que nuestro destino se encuentra en lo suprasensible.

\section{La consagración de lo sublime tecnológico}

A fin de percibir la afinidad de las dualidades entre las cuales se escinde la ética kantiana, con el funcionamiento igualmente dualista de la estética, convendría remitirnos al parentesco entre la antítesis Lust/Unlust y la alternancia entre las fuerzas de maximización (vinculadas a la "imaginación productora") y de restricción de la actividad (relacionadas con las propiedades legislativas del entendimiento); si se tiene en cuenta que el principio de placer y maximización de energía exige la perpetuación del estado presente, mientras que el Unlust requiere la sustitución de la situación actual por una aspiración utópica, podrá comprenderse que placer y displacer se asienten, respectivamente, sobre una situación de hecho o sobre una imposición legislativa; dicho de otro modo: sobre una descripción o una pres- 
cripción. Sin embargo, el Unlust no es señalado por Kant como fuerza exclusivamente destructora, ya que a menudo una representación sensualmente repulsiva favorece la dominación de las inclinaciones y se aviene, por ende, con los más altos designios de la razón: aquí, la obstrucción de los impulsos vitales redunda mediatamente en una liberación aun mayor de la energía, solo que esta no corresponde ya al ámbito de la sensibilidad corpórea, sino al de la racionalidad. Este es el caso de lo sublime; la Hemmung, la inhibición momentánea de las fuerzas de la vida, ante cuya comparecencia se suspende la sensación de pertenencia al mundo tangible, es la efímera vía para que precipitadamente se descargue la Ergießung: una efusión de energía que, al no proceder de un acuerdo de la facultad imaginativa con el entendimiento, sino con la razón, posee una naturaleza diversa de la inmediata Belebung que se deriva de la contemplación de lo bello. Se comprenderá cuál es la base de sustento para la advertencia kantiana de que el bien moral sea representado como sublime: la vitalidad que proporciona el cumplimiento de los desagradables preceptos morales en el campo de la ética es proporcional a la que recibe el espectador del arte y de la naturaleza elevada; en ambos casos, la vigorización resulta de la repulsa de la realidad material y de la trascendencia hacia lo suprasensible.

Aquí queda expuesta en toda su crudeza la índole del idealismo kantiano. Pero es pertinente insistir sobre el desgarramiento entre descripción y prescripción: pudimos ver de qué manera la ética kantiana surge de una crisis en la que el origen de los valores se ha tornado indeterminable; si es desatinado pretender deducir el Sollen de la praxis concreta (puesto que los principios a priori no deberían transigir con la realidad fenoménica) entonces la violencia legislativa de lo posible se ubicará por encima de lo real: la comunidad utópica prevalecerá sobre la empírica. Otro tanto ocurre en el interior de la estética: si el carácter inmediatamente necesario del Geschmacksurteil procede de la efectiva universalidad del sensus communis, lo sublime exige, en cambio, una mediación: aquella que impone la ley mo$\mathrm{ral}^{22}$; en esa medida, la sublimidad supera a la belleza de la misma manera en

22 “[...]le sentiment sublime fait certes appel aussi à une participation universelle [...] Mais cet appel ne peut pas être immédiat comme dans le goût. L'exigence 
que lo posible - lo "prescriptivo" - supera a lo vigente. Tengamos en cuenta que lo sublime requiere de la cultura moral, y que esta no es jamás para Kant una realidad de hecho, sino solo una meta a lograr; de ahí la certidumbre de Peña Aguado de que en lo sublime se condensa la visión (estética) kantiana de una Gemeinschaft utópica, contrapuesta a la comunidad real en que se asienta lo bello:

Die Allgemeingültigkeit des Erhabenen kann nicht wie im Falle des Schönen vorausgesetzt werden. So wie die Lust am Erhabenen nur mittelbar ist, so wäre das Gefühl des Erhabenen nur unter bestimmten kulturellen Bedingungen allgemeingültig. Kultur ist im Sinne Kants ein Prozeß und gleichzeitig ein Ziel, das durch die Entfaltung der Empfänglichkeit zu erreichen ist. Von daher käme der Anspruch auf Allgemeingültigkeit einer Denkweise wie der des Erhabenen an Idealität gleich. Während die Allgemeingültigkeit des Schönen einen sensus communis voraussetzt und die Bildung einer Gemeinschaft impliziert, ist die Allgemeingültigkeit des Erhabenen im Bezug auf diese Gemeinschaft nur als Utopie denkbar (PEÑA Aguado 1994: 63).

La sublimidad, esa experiencia que, al decir de Kant, hunde sus raíces en la naturaleza humana, participa de un relativo elitismo ${ }^{23}$; esta paradoja es aparente: el democrático gesto con el que Kant proclama la universalidad del juicio estético sublime, contrasta con la aseveración de que la sublimidad requiere de un cierto desarrollo de las ideas morales, sin el cual la experiencia estética se mantendría petrificada en un salvaje temor. Esta ambigüedad es propia de una sociedad que recusa la violencia desnuda para colocar en su lugar una violencia encubierta, internalizada; la Kultur requerida por Kant

d'universalité qui est propre au sublime passe 'par une médiation, vermittelst, celle de la loi morale, des moralischen Gesetzes' [...]” (LyOtard 1991: 271).

23 Cfr. "[...] Was den Einzelnen betrifft und obwohl Kant sich nicht expressis verbis dazu äußert, gewinnt man im Verlauf seiner Darstellung den Eindruck, daß das Erhabene ein sehr elitäres Gefühl ist. Elitär, weil das Gefühl des Erhabenen nicht jedem (und erst recht nicht jeder) zugänglich ist, obwohl dieses Gefühl sich auf eine gemeinsame Gemütsgrundlage beziehen läßt” (PeÑa Aguado 1994: 64). 
coincide con aquella domesticación del sujeto a la que hemos aludido. Una cierta cultura moral es, precisamente, lo que requiere el individuo para no experimentar horror ante ese poder, inmaterial e incorpóreo, que lo acosa y oprime: a través de la educación no se aprende solo a conjurar el temor frente a la inquietante naturaleza - a la que el hombre no ilustrado observaba con reverencia y espanto -, sino también a contemplar con noble devoción la aun más temible potencia del espíritu puro: por tanto, la resistencia que la humanidad ilustrada opone ante el poder indómito de lo salvaje, encuentra su correlato en el respeto que le inspiran las inmateriales leyes abstractas. El avance de la cultura no coincide solo, para Kant, con el progreso de la opresión de lo natural, sino además con el desarrollo de un poder racional, abstracto y autónomo, que se enfrenta a los hombres con un autoridad no menos tiránica que la de la naturaleza mítica, pero que reclama una aceptación inobjetable, puesto que sus determinaciones se hallarían inscriptas en el interior del espíritu humano. El hecho de que Kant afirme que el desarrollo de la cultura coincide con el surgimiento de un Dios sobrenatural ${ }^{24}$, es otra prueba del paralelismo que existe entre el progreso de la cultura y el triunfo de la abstracción. Es sugestivo que, para Kant, la identificación entre logos y divinidad constituya un logro de las Luces, pero también lo es que la representación de una divinidad incorpórea y, por lo tanto, irrepresentable, provea la más alta instancia de sublimidad. Todo pone de manifiesto una serie de paralelismos: el impulso que ha permitido a la fe monoteísta derrotar a las religiones antropomórficas precedentes es análogo al que ha favorecido el triunfo de la razón por sobre la indigna naturaleza, y al que permite a la impresentable sublimidad trascender al Geschmacksurteil. En los tres casos se advierte la presencia de una potencia invisible e imponente, de un poder suprasensible que, a diferencia de la finitud natural, tiende incesantemente hacia lo ilimitado.

El carácter dinámico de este poder, unido a su incorporeidad y abstracción, nos remite a uno de los puntos principales del análisis de la filoso-

24 "Kant sees the development of culture as accompanied by the emergence of a sublime God, an idea of divinity resting not on the might of nature, but on the dominion of the supernatural" (CAYGILL 1989: 346). 
fía kantiana emprendido por Lukács en Geschicbte und Klassenbewnßtsein, a saber: la equiparación del formalismo kantiano con la tendencia, propia de la sociedad burguesa, a elevar la categoría de cantidad al rango de categoría representativa de la modernidad. La supresión de las diferencias cualitativas bajo la universal identidad del concepto es, en el terreno del pensamiento, la otra cara del proceso por el cual la generalización del tráfico mercantil conduce a la eliminación de los caracteres específicos de los objetos - su valor de uso - y al establecimiento de la identidad abstracta, cuantitativamente mensurable y objetivada en el valor de cambio; del mismo modo, la institución de las máximas morales abstractas y el acallamiento de los deseos individuales, acompañan y refuerzan la evolución de una estructura social que exige, a la vez, el sometimiento de la naturaleza externa bajo el imperativo del cálculo racional y la supeditación de los instintos a la expectativa de la utilidad futura. En todos los casos, la dinámica de la ratio ignora todo límite y medida: el crecimiento cuantitativo de la abstracción convierte en insuficiente toda estimación de magnitudes; por una curiosa ironía, resulta, pues, que la razón - cuya actividad consiste en reducir la plenitud de la realidad a lo abstractamente mensurable - supera ella misma todo cálculo comprehensivo y se proyecta a lo infinito. El joven Marx, quien advirtió la "mala infinitud" del capitalismo moderno, describió el avance puramente cuantitativo del capital en términos próximos a la sublimidad kantiana: "Die Quantität des Geldes wird immer mehr seine einzige mächtige Eigenschaft; wie es alles Wesen auf seine Abstraktion reduziert, so reduziert es sich in seiner eignen Bewegung als quantitatives Wesen. Die Maßlosigkeit und Unmäßigkeit wird sein wahres Maß” (MARx 1956: 40, 547). La poderosa (y dominante) enérgeia de la razón instrumental, objetivada en la expansión incontenible de las fuerzas productivas y, consiguientemente, del capital, constituye la expresión más cabal de lo sublime, tal como es presentado en la estética kantiana. En este sentido, es apropiada la asociación que establece Lyotard entre lo sublime y la era de la técnica ${ }^{25}$; y la formulación es doblemente acertada

25 'Heidegger dira qu'avec la technique, l'être se donne comme 'fonds', qu'on a sous la main, dans quoi l'on puise, sans avoir à l'entendre. Mutatis mutandis, le Gebrauch kantien annonce un motif analogue, la dénaturation de l'être qui rend 
porque, al delatar la correspondencia entre la sublimidad kantiana y el animus de los tiempos más recientes, permite inferir que la filosofía de Kant es predecesora y, hasta cierto punto, también coetánea del despliegue de las fuerzas productivas que ha acompañado al progreso de la sociedad burguesa ${ }^{26}$.

La Formlosigkeit de la sublimidad kantiana es, entonces, reflejo fiel de la ausencia de forma correspondiente a esa segunda naturaleza que, aunque creada por los hombres, no es sin embargo menos opresiva que la mítica naturaleza primera. El hecho de que la segunda naturaleza sea una realidad abstracta y convencional, dominada por leyes a las que ha sido sustraído todo anclaje en la comunidad, explica que la sociedad moderna aparezca, ante los ojos del individuo, como algo extraño, inbumano. La segunda naturaleza es, ante todo, obra de la Kultur y, por ende, el fruto del sojuzgamiento y aniquilación de la naturaleza primera. Se impone sobre la sensibilidad como una realidad inabarcable y hostil, que por su carencia de forma escapa a todos los esfuerzos humanos de conceptualización. Los resultados de esta pérdida de la comprensibilidad inmediata son ostensibles y no requieren de especificaciones; bastaría con mencionar algunas de sus revelaciones: pensemos en los efectos destructores que ha ejercido sobre la consciencia del individuo la liquidación de las llamadas sociedades cerradas y la globalización de la economía; pensemos, igualmente, en la estructura de las modernas urbes, cuya estructura inconmensurable, informe, se sustrae a los esfuerzos de las facultades perceptivas. Puede parecer llamativo que Kant, desde una Alemania todavía provinciana, haya podido establecer una analogía para procesos que comenzaban a desplegarse en las naciones más desarrolladas; pero, ¿no ha encontrado el joven Lukács en el pensamiento kantiano la objetivación más alta de las antinomias del cosificado pensamiento burgués? En todo caso, en esta cualidad visionaria del filósofo debemos ver los gérmenes de la posterior apropiación postmoderna de su filosofía. No es casual que Jameson, a la hora de referirse a la ausencia de

caduc le poème, et qui permet le moyen. - Je ne poursuis pas cette ligne grossièrement esquissée mais qui conduirait peut-être à l'intelligence de l'affinité des esthétiques sublimes avec l'époque de la technique" (LyOtARD 1991: 92).

26 Pero Lyotard no arriba a esta conclusión. 
forma de los hiperespacios urbanos - engendrados en su forma plena durante el capitalismo tardío - recurra a la categoría de sublimidad, y que ponga el acento en la esencial desorientación del hombre contemporáneo:

$\bigcirc$

吕

[the] post-modern hyperspace [...] has finally succeeded in transcending the capacities of the individual human body to locate itself, to organize its immediate surroundings perceptually, and cognitively to map its position in a mappable external world. It may now be suggested that this alarming disjunction point between the body and its built environment [...] can itself stand as the symbol and analogon of that even sharper dilemma which is the incapacity of our minds, at least at present, to map the great global multinational and decentered communicational network in which we find ourselves caught as individual subjects (JAMESON 1991: 44).

Puede verse que las objetivaciones producidas durante "la era de la técnica", aun cuando admitan ser aprehendidas parcialmente, se sustraen (como las representaciones sublimes o, mejor aun, como la "totalidad inteligible" de la razón) a todo esfuerzo comprehensivo. En todos estos casos vemos reproducida la lógica de la sociedad mercantil: la correlación entre el detalle regulado y el todo casual, entre la legislación de lo singular y la relativa irracionalidad de las estructuras globales. Pero si las instituciones sociales se presentan ante los ojos del individuo como extrañas e inhumanas - positivas, en el sentido de Hegel -, tenemos que ver en ello un contrasentido, puesto que la segunda naturaleza es producto de la actividad del hombre; por ende, la aparente contingencia e inorganicidad que asume ante nuestros ojos la realidad social, debería ser desmitificada como ilusoria:

[An] objection might be made to the reality of this contingence in modern life: it is contingency, it might be said, in appearance only. In fact, all such apparently inhuman institutions and things are intensely human in their origin. Never has the world been so completely humanized as in industrial times; never has so much of the individual's environment been the result, not of blind natural forces, but of human history itself (JAMESON 1971: 168). 
La respuesta que Jameson (apoyándose en Lukács) propone frente a la cosificadora abstracción de la sociedad moderna, es, en el terreno del arte, la defensa de la concreción - lo que no puede menos que evocar las sugerencias de Baumgarten -. Es a través de una representación de la realidad en términos humanos que el arte conjura la Formlosigkeit de la sociedad burguesa y de sus correlatos científicos y filosóficos; la salvación no se obtiene subsumiendo a la sociedad existente bajo una prescripción abstracta, sino partiendo de los hombres concretos y la experiencia cotidiana. Podemos deducir de esto una crítica oblicua a la filosofía kantiana, por cuanto de aquí se infiere que solo reanudando el contacto con la tradición y la experiencia colectiva podrá ponerse un límite a la expansión de la ratio.

Pero, por otro lado, sería insensato pensar que en la belleza kantiana reside una legítima alternativa frente a las contradicciones de la sublimidad. En el Geschmacksurteil, la apelación al equilibrio y la medida evitaba la solución trágica: naturaleza y racionalidad podían llegar a un acuerdo, en tanto el entendimiento creyese reconocer en la realidad fenoménica las huellas de una sumisión dócil y espontánea: por eso Eagleton compara a la belleza con la hegemonía, puesto que la tirantez entre legislación y gusto, así como la escisión entre espíritu y naturaleza, son por ella momentáneamente suspendidas. La violencia originaria queda disimulada; estas observaciones sugieren examinar al juicio de gusto con mayor escepticismo, ya que el acuerdo entre naturaleza y entendimiento no es producto de una espontánea armonía, sino resultado de que los desgarramientos y tensiones han sido disimulados, y de que esa violencia enmascarada de normalidad implica igualmente el sojuzgamiento de la naturaleza. No es casual que hegemonía y belleza coincidan en su apelación al sensus communis ${ }^{27}$, ya que ambas reclaman una aprobación inmediata que no puede admitir dudas, puesto que estas podrían disolver la necesaria apariencia de normalidad. En lo sublime prima, por el contrario, el diferendo, y las mutuas divergencias y hostilidades entre naturaleza y razón son llevadas hasta una tensión extre-

27 Raymond Williams diferencia a la begemonía de la ideología, por cuanto la primera "[...] is seen to depend for its hold not only on its expression of the interests of a ruling class but also on its acceptance as 'normal reality' or 'commonsense' by those in practice subordinated to it" (Williams 1984: 145, s.v. "Hegemony”). 
ma: a la pasión clasificatoria del entendimiento humano, que busca reducir a categorías abstractas la diversidad de la naturaleza, esta puede oponer su propia magnificencia, ante la cual el hombre teme perderse como en un abismo; sin embargo, a la Hemmung desalentadora la razón enfrenta la propia infinitud, ante cuya violencia no puede la naturaleza presentar nada análogo. Y hay en esto una medida de verdad, puesto que nada de lo que pueda encontrarse en la naturaleza indómita iguala a la barbarie de la ratio instrumental. Aquí se pone de manifiesto de qué manera la estética de Kant representa el punto de transición hacia lo sublime de la técnica: el momento en que la "antiestética" magnanimitas de la razón supera a la que era propia de los escenarios salvajes. La satisfacción que proporciona el acuerdo entre imaginación y racionalidad no posee ningún poder desmitificador: es solo la laudatio entonada por la imaginación para celebrar la instrumentalización de lo natural.

\section{Referencias bibliográficas}

Adorno, Th. W. Gesammelte Schriften. Ed. de Rolf Tiedemann en colab. con Gretel Adorno, Susan Buck-Morss y Klaus Schultz. 20 vv. Darmstadt, Wissenschaftliche Buchgesellschaft 1998.

Burke, E. On Taste. On the Sublime and Beautiful. Reflections on the French Revolution. New York, Collier \& Son ("The Harvard Classics") 1963.

Caygill, H. Art of Judgment. Oxford, Basil Blackwell 1989.

Eagleton, T. The ideology of the aesthetic. Oxford, Basil Blackwell 1991.

Eagleton, T. Heathcliff and the Great Hunger. London/New York, Verso 1995.

Hegel, G. W.F. Werke in zwanzig Bänden. Red.: Eva Moldenhauer y Karl Markus Michel. Frankfurt a. M., Suhrkamp 1971.

Horkheimer, M. "Egoismus und Freiheitsbewegung". In: Traditionelle und kritische Theorie. Vier Aufsätz̨e. Frankfurt a. M./Hamburg, Fischer 1970, 95-161.

Jameson, F. Marxism and Form. Towards a Dialectical Criticism. Princeton, Princeton U. P. 1971. 
Jameson, F. Postmodernism or, the cultural logic of late capitalism. Durham, Duke U. P. 1991.

Kant, I. Kritik der Urteilskraft. Ed. de Karl Vorländer. Hamburg, Felix Meiner 1990.

Larthomas, J.-P. De Shaftesbury à Kant. Paris, Didier Érudition 1985.

Lentricchia, F. After the New Criticism. Chicago, The University of Chicago Press 1980.

LukÁcs, G. Der junge Hegel. Über die Bequiehungen von Dialektike und Ökonomie. 2 Bd. Frankfurt a. M., Suhrkamp 1973.

Lyotard, J.-F. "L'intérêt du sublime". In: Nancy, J.-L. (ed.), Du Sublime. Paris, Berlin (“L'Extrême Contemporain”) 1988, 149-178.

Lyotard, J.-F. Leçons sur l'Analytique du sublime. Paris, Galilée 1991.

MacIntyre, A. After Virtue: a Study in Moral Theory. London, Notre Dame 1981.

Marcuse, H. Eros and Civilization. A Philosophical Inquiry into Freud. London, Abacus 1973.

Marx K. y Engels, F. Werke. ed. por el Institut für Marxismus-Leninismus beim ZK der SED. 43 Bd. Berlin, Dietz-Verlag 1956 ss.

Peña Aguado, M. I. Ästhetik des Erhabenen (Burke, Kant, Adorno, Lyotard). Wien, Passagen 1994.

Trilling, L. "Manners, Morals, and the Novel”. In: Scholes, R. (ed.), Approaches to the novel. Materials for a Poetics. San Francisco, Chandler 1961, 231-246.

Williams, R. Culture and Society (1780-1950). London, Chatto \& Windus 1960.

Williams, R. Keywords. A vocabulary of culture and society. London, Fontana 1984. 